\title{
La confiance dans les relations interentreprises : antécédents, retombées, mesures et découvertes récentes ${ }^{1}$
}

\author{
Marc-Antoine Vachon et Lova Rajaobelina \\ ESG-UQAM
}

\section{INTRODUCTION}

\section{Bien qu'il soit établi depuis un moment que la confiance doit être une priorité dans les relations, la confusion conceptuelle, opérationnelle et méthodologique autour du concept demeure bien réelle.}

Bien qu'il soit établi depuis un moment que la confiance doit être une priorité dans les relations, la confusion conceptuelle, opérationnelle et méthodologique autour du concept demeure bien réelle ${ }^{2}$. Plusieurs auteurs ont fait allusion à cette confusion notamment en réclamant davantage de clarté conceptuelle ${ }^{3}$ et une meilleure différenciation des antécédents, des composantes et des retombées de la confiance ${ }^{4}$. Ces constats démontrent la nécessité de recherches théoriques sur la confiance avant de procéder à davantage d'évaluations empiriques du construit.

La confiance est un élément fondamental des relations interentreprises. Elle a été étudiée sous plusieurs angles et dans plusieurs contextes différents. Certains auteurs ont recensé jusqu'à 22 dimensions associées à la composition de la confiance dans la littérature ${ }^{5}$. L'extensivité des recherches académiques a rendu difficile de bien saisir la réelle signification du construit et son rôle dans les relations.

Les antécédents et les retombées de la confiance ont été classifiés selon différentes typologies et ont fait l'objet de quelques méta-analyses ${ }^{3,6}$. Les résultats demeurent toutefois mitigés, particulièrement en raison du manque de clarté conceptuelle. De plus, les mesures utilisées dans les études semblent avoir une validité de contenu discutable. Par exemple, lors d'une analyse utilisant des études portant sur la confiance interentreprises, la majorité d'entre elles (9/15) n'avaient pas effectué de prétest des questionnaires. Cette lacune méthodologique laisse place au doute quant à la validité des résultats.

Cet article a donc pour objectif de proposer une nouvelle typologie des antécédents et des retombées de la confiance interentreprises dans un contexte de relation acheteur-vendeur et de proposer une mesure adéquate de la confiance.

Tout d'abord, le construit de la confiance est discuté et la conceptualisation préconisée est argumentée. Par la suite, les antécédents et les retombées recensés dans la littérature sont analysés et regroupés sous des catégories qui se retrouvent dans le cadre conceptuel. De plus, une proposition de mesures de la confiance, issues d'une analyse littéraire, est présentée afin d'améliorer la fidélité et la validité des instruments de mesure dans l'étude du construit. Les implications de l'article sont ensuite discutées et ses limites sont exposées puis liées à des avenues de recherches futures. Cet article devrait contribuer à clarifier le concept de confiance, à faciliter sa mesure et à augmenter la variance expliquée dans les recherches impliquant ce construit.

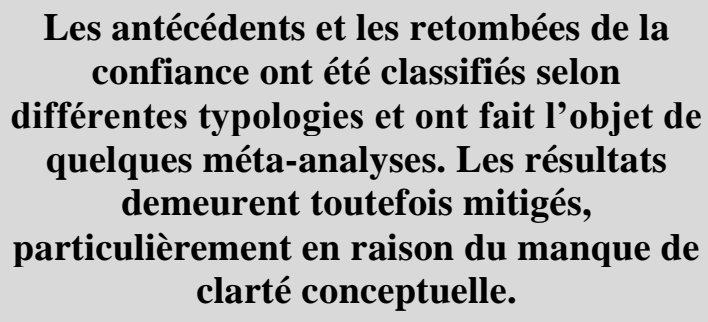

Les antécédents et les retombées de la confiance ont été classifiés selon différentes typologies et ont fait l'objet de quelques méta-analyses. Les résultats demeurent toutefois mitigés, particulièrement en raison du manque de clarté conceptuelle. 


\section{LES FONDEMENTS}

La confiance a été étudiée dans plusieurs disciplines, dont la psychologie, la sociologie, l'économie, le management et le marketing. La complexité du concept de confiance provient d'entrée de jeu de ses bases fondamentales. Trois facettes expliqueraient la formation de la confiance: une cognitive, une affective et une comportementale ${ }^{7}$.

La facette cognitive se focalise sur des fondements rationnels comme les caractéristiques du marchand, par exemple la compétence et la fiabilité. La confiance basée sur la cognition ou sur la connaissance et la confiance technique sont autant de termes utilisés pour cerner cette première facette. Il s'agit ici d'une évaluation rationnelle.

La facette affective inclut différentes formes de la confiance comme la confiance émotionnelle, la confiance basée sur l'affect ou sur l'identification, la confiance relationnelle, la confiance interpersonnelle et la confiance morale. Cette facette est souvent matérialisée par la bienveillance. Il s'agit ici d'une évaluation émotionnelle.
La facette comportementale se traduit par des façons d'agir qui sont dictées par la confiance. Il est important de distinguer l'intention comportementale (ex. : intention ou volonté de suivre les conseils) du comportement lui-même (ex. : l'achat). À propos du rôle du comportement au sein de la confiance, on recense deux approches. L'approche comportementale se résume par deux concepts, soient les attentes face aux comportements de la cible (ex. : l'autre entreprise) et la vulnérabilité qu'une source (ex. : notre entreprise) est prête à accepter. Cette approche considère le comportement comme faisant partie du construit de la confiance. L'approche psychologique perçoit la confiance comme un indicateur du comportement futur. Elle est donc en amont de l'intention et a été traduite dans la littérature par une attente, une présomption ou une croyance ${ }^{8}$. Plusieurs études récentes ont préféré l'approche psychologique.

Bref, la confiance est un mélange de pensée rationnelle et d'émotions qui se traduisent en comportements tel que présenté à la figure 1 . Elle laisse place à plusieurs interprétations de la part des chercheurs, ce qui contribue à la complexité du construit.

\section{Figure 1 - Formation de la confiance}

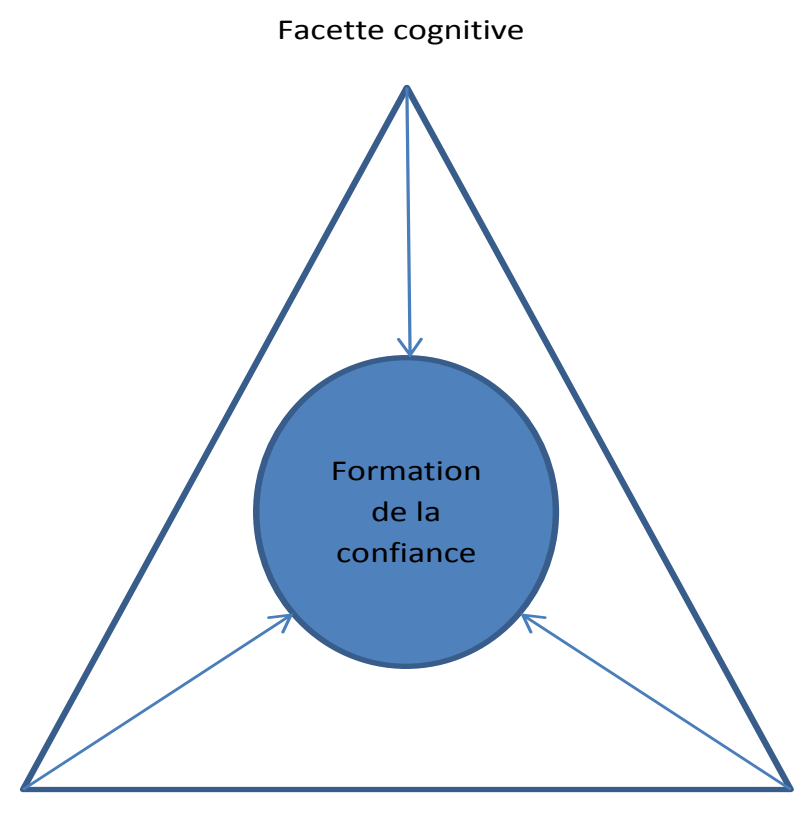

Facette

comportemantale

Facette affective 


\section{VERS UNE DÉFINITION}

La confusion entourant le concept de la confiance se poursuit dans la multiplicité de ses définitions. Elles n'utilisent pas toujours les mêmes composantes. Certains chercheurs n'incluent même pas les dimensions qu'ils étudient dans leur définition ${ }^{2}$. Certains voient la confiance comme étant tout simplement le respect des promesses et des obligations, d'autres comme le degré auquel un client croit qu'il peut se fier que le vendeur agira dans son meilleur intérêt.

Mais la littérature a démontré qu'une conception bidimensionnelle a été dominante dans les années 80 et 90 qui incluait surtout l'honnêteté (fiabilité et respect des promesses) et la bienveillance (intérêt envers le bien de l'autre) mais parfois la compétence et la bienveillance ou encore l'intégrité et l'altruisme. Par exemple, pour certains auteurs, la confiance est la perception de crédibilité et de bienveillance qu'a une cible ${ }^{9}$. La crédibilité consiste à croire que l'autre effectuera le travail efficacement et qu'il est fiable. La bienveillance est ici la propension de quelqu'un à veiller au bien de l'autre et sa motivation à atteindre des gains mutuels. Une autre conception intéressante préfère considérer la fiabilité et l'intégrité du partenaire, tenant compte des qualités associées à la confiance relevées dans la littérature : la constance, la compétence, l'honnêteté, être équitable, responsable, aidant et bienveillant ${ }^{10}$.

Ces conceptions bidimensionnelles révèlent un certain chevauchement conceptuel. La crédibilité rassemble le fait d'être capable d'effectuer le travail et l'intention de respecter ses engagements, deux concepts qui semblent distincts. L'intégrité comprend le respect des promesses et la bienveillance qui est associée à la réaction accommodante en cas de situation non prévue.

Cet empiètement conceptuel laisse place à d'autres conceptualisations de la confiance telle que celle à quatre dimensions. Par exemple, certains en sont venus à inclure la compétence (connaissance et donne de bonnes informations), la responsabilité (qui est opérationnalisée comme de la bienveillance), la « dependability» (qui est opérationnalisée comme de la fiabilité, être digne de confiance) et l'agréabilité (qui se rapporte aux relations interpersonnelles).

Dans un contexte web, des auteurs prônent une confiance composée de quatre dimensions : la compétence (aptitude technique et expertise), l'intégrité-facette 1 (honorer ses engagements), l'intégrité-facette 2 (sécurité des échanges d'information sur le web) et l'intérêt pour régler les problèmes $^{8}$. Cette dernière dimension est un mélange de deux dimensions qu'ils croyaient distinctes : la bienveillance (attention pour les intérêts de l'autre) et l'orientation à résoudre les problèmes. Leur explication à cette fusion provient de la similarité conceptuelle entre le fait de porter attention aux intérêts de l'autre et de la propension à régler ces problèmes. De plus, l'absence de contacts personnels en contexte web rend difficile l'évaluation de la bienveillance.

Une autre conception de la confiance, intéressante pour sa démarcation plus claire entre les dimensions tout en faisant référence à suffisamment d'éléments de la confiance pour la considérer comme représentative du construit, est celle à trois dimensions. Par exemple, certains avancent trois composantes de la confiance: l'attention portée aux intérêts de la cible (dependability), le fait de donner un bon rendement, ce qui était prévu (predictability) et finalement le fait de croire que l'autre n'adoptera pas un comportement opportuniste (faith). Cette tendance tridimensionnelle est de plus en plus présente dans la littérature. Les dimensions qui en ressortent sont la compétence (expertise), l'intégrité (respect des promesses) et la bienveillance (attention aux besoins de l'autre).

Le présent article adhère à cette conception tridimensionnelle de la confiance. D'ailleurs, la définition de la confiance proposée ici intègre les trois dimensions. Avant de présenter la définition, il est important de clarifier que la confiance est une croyance basée sur des perceptions. Le terme croyance est utilisé car il n'y a pas de certitude quant à l'évaluation des dimensions. La source évalue sa croyance à l'effet que la cible est bienveillante, compétente et intègre, mais il n'y a 
pas de certitude à cet égard. Les perceptions sont importantes car une fois de plus, l'évaluation de la source se base sur certaines références. La perception de ces références déterminera la croyance à l'égard des dimensions de la confiance. Par exemple, la perception d'une source à l'égard de la franchise d'une cible lui permettra de croire qu'elle est intègre et par le fait même, digne de confiance. C'est suite à une évaluation des composantes de la confiance qu'une source peut se prononcer sur son niveau de confiance et ensuite adapter son répertoire comportemental. Ainsi, la confiance se définit comme un ensemble de croyances basées sur la perception d'une source quant à la bienveillance, la compétence et l'intégrité d'une cible.

\section{La confiance se définit comme un ensemble de croyances basées sur la perception d'une source quant à la bienveillance, la compétence et l'intégrité d'une cible.}

D'abord, La bienveillance rapporte à la croyance que la cible a les intérêts de la source à cœur et

\section{LES DIFFÉRENTS TYPES DE CONFIANCE}

La confiance peut être analysée sous différents angles, notamment au niveau des individus, des dyades, des groupes, des réseaux ou alliances, des systèmes, des entreprises, des marques, ou des produits. Généralement, la littérature dénote principalement deux cibles envers lesquelles la confiance est considérée : une personne (soit le représentant de l'entreprise - souvent le vendeur) ou une entreprise.

Cette dichotomie est nécessaire puisque les bases sont différentes selon le niveau émotionnel de la relation. Le transfert de confiance de l'entreprise à son représentant est à double sens. La confiance qu'un acheteur porte à une entreprise peut se transférer au vendeur devant lui. L'inverse peut être vrai où un acheteur faisant confiance à un vendeur peut accorder sa confiance à l'entreprise que ce dernier représente. Par ailleurs, il a été démontré que la confiance envers un représentant ${ }^{11}$ et la confiance envers une entreprise jouent des rôles différents dans la relation. Cet article préconise qu'elle sera accommodante en cas de situations problématiques. La bienveillance est sans doute la dimension de la confiance la plus souvent considérée ne serait-ce qu'implicitement. Elle a déjà été appelée "dependability », altruisme ou encore intérêt pour les problèmes. Cette dimension peut sembler comporter deux facteurs différents en "avoir l'intérêt de la source à cœur» et «accommodante en cas de problème ». Toutefois, certains auteurs en sont venus à la conclusion qu'ils formaient bel et bien une seule dimension puisque résoudre des problèmes va de soi lorsqu'on a l'intérêt du client à cœur. Ensuite, la compétence réfère à la croyance que la cible possède la capacité de réalisation du travail, l'expertise suffisante pour son accomplissement. Cette composante rassemble des terminologies telles que crédibilité, fiabilité ou «predictability». Enfin, l'intégrité se rapporte à la croyance que l'autre respectera ses promesses et que les informations divulguées sont véridiques. L'intégrité a souvent été mélangée conceptuellement à la compétence ou parfois à la bienveillance sous des appellations telles qu'honnêteté ou crédibilité.

donc l'intégration au cadre conceptuel des deux types de confiance les plus reconnus soit la confiance envers l'entreprise et la confiance envers le représentant.

Il est à noter que la distinction entre les définitions de la confiance interpersonnelle, organisationnelle et interorganisationnelle est imperceptible. Dans la majorité des cas, la voix passive est utilisée dans les définitions pour dénommer l'autre partie comme « le partenaire» ou « la cible», ce qui laisse place à une autre partie de nature diverse qu'elle soit une personne ou une entreprise. Certains auteurs suggèrent d'ailleurs que tous les types de confiance devraient partager des concepts communs afin de faciliter le consensus sur le construit. La définition de la confiance proposée dans cet article respecte ce constat et peut donc être attribuée à l'un ou l'autre des types de confiance puisque la source et la cible peuvent être de natures diverses. La différence est davantage observable au niveau des antécédents de chacun. 


\section{LES ANTÉCÉDENTS}

De nombreux antécédents, soit des facteurs qui favorisent la confiance, ont pu être recensés dans la littérature. En fait, plusieurs ont des appellations différentes tout en demeurant conceptuellement très proches. Certains auteurs ont tenté de les catégoriser. Une étude a tout d'abord classé les antécédents et les conséquences de la confiance à l'intérieur de huit catégories. Suite à une méta-analyse, cinq catégories d'antécédents en sont ressorties : l'incertitude environnementale (associée au risque perçu), la dépendance, l'utilisation du pouvoir coercitif, la communication et les retombées économiques ${ }^{6}$. Toutefois, la validité de contenu de certaines catégories demeure à démontrer. Une autre étude réalisée sous forme de méta-analyse a classifié les antécédents de la confiance interpersonnelle entre acheteurs et vendeurs. Les catégories s'intitulent: la bienveillance du vendeur, la compétence du vendeur, l'agréabilité/similarité $\mathrm{du}$ vendeur, les techniques de vente du vendeur, l'expérience avec le vendeur et les déterminants associés à la firme du vendeur ${ }^{3}$. Cette conception amène une vision différente des composantes de la confiance puisque la compétence et la bienveillance sont considérées ici comme des antécédents et non des composantes.

Ces méta-analyses concernent des types de confiance différents telles que la confiance envers une entreprise et la confiance envers un représentant. Puisque la première étude n'a pas permis d'y rattacher d'importants antécédents éprouvés dans la littérature et que leur modèle explique une faible variance du construit, une modification de sa typologie s'impose. Le modèle de la deuxième étude intègre des composantes de la confiance au sein des antécédents et comporte quelques éléments de la confiance envers l'entreprise.

Puisqu'il a été démontré que la confiance envers un représentant et la confiance envers une entreprise jouent des rôles différents dans la relation et que des mesures adaptées à chaque catégorie doivent être utilisées, il serait important de respecter cette différence dans l'élaboration d'une typologie intégratrice. Cependant, certains déterminants de la confiance sont tout aussi attribuables à une entreprise qu'à un représentant. Les antécédents doivent donc être divisés parmi les deux grands types de confiance (envers un représentant et envers une entreprise) ainsi qu'une troisième section qui s'adresse aux deux types à la fois.

Le cadre conceptuel de la figure 2 intègre les antécédents et les retombées de la confiance ${ }^{12}$. Elle est présentée comme un construit unidimensionnel puisque l'utilité d'isoler l'effet de chaque dimension est discutable, ne menant pas à des recommandations managériales plus utiles.

Figure 2 - Cadre conceptuel

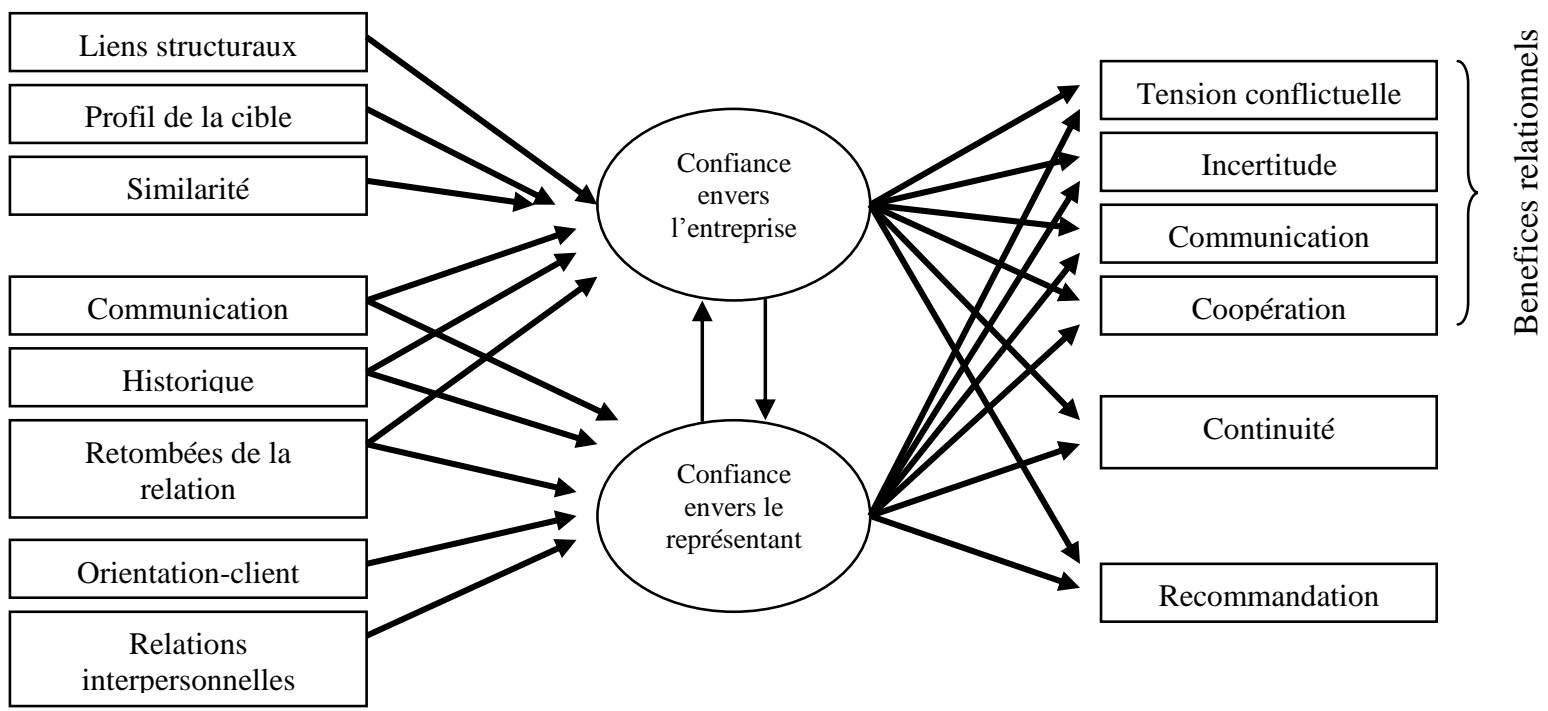


Les trois premières catégories se rapportent à la confiance envers une entreprise. Les Liens structuraux concernent les liens tangibles qui unissent les entreprises comme les adaptations spécifiques réalisées dans le but de faciliter la relation ${ }^{9}$. Ces liens, notamment par l'investissement, démontrent que l'entreprise tient à la relation et qu'elle est prête à faire des sacrifices $^{13}$. Le Profil de la cible concerne sa taille et sa réputation. Une taille plus importante supposerait que l'entreprise a dû entrer en relation avec plusieurs entreprises et qu'au fil du temps, elle a connu du succès9. D'autres entreprises pourraient être rassurées de cette expérience. L'effet de la réputation s'effectue en démontrant qu'au fil du temps, le comportement de la cible a été constant ${ }^{13}$.

La Similarité entre entreprises réfère à la congruence des buts recherchés par les entreprises en présence, le fait qu'elles partagent les mêmes valeurs ainsi que la balance du pouvoir au sein de la relation. Un consensus entourant les objectifs de croissance est susceptible de diminuer les conflits et ainsi favoriser la formation de la confiance. Pour ce qui est des valeurs partagées, en ayant une perspective similaire sur ce qui est approprié ou non ou encore sur ce qui est important ou non, la formation de la confiance sera facilitée ${ }^{10}$. De plus, si une partie peut compter sur un nombre plus important d'alternatives qu'une autre ou si elle peut contraindre l'autre à faire quelque chose, elle a le plus de pouvoir. Cette inégalité peut mener à des abus et à des conséquences négatives pour la confiance. Ainsi, un pouvoir réparti de manière inégale pourrait rendre davantage difficile la formation de la confiance notamment en ce qui a trait aux intentions du plus puissant en cas d'imprévus.

Les trois prochaines catégories se rapportent aussi bien à la confiance envers une entreprise qu'envers un représentant. La Communication se veut un échange bidirectionnel d'information ${ }^{14}$. Une bonne communication joue un rôle prépondérant dans la formation de la confiance, par exemple en facilitant le règlement des conflits, en favorisant la transparence ou encore l'alignement des perceptions et des attentes.
Les Retombées de la relation sont reconnues pour avoir l'effet le plus prononcé sur le niveau de confiance ${ }^{6}$. Cette catégorie rassemble principalement la perception de qualité et la satisfaction, deux concepts étroitement liés qui se rapportent à une expérience antérieure. La perception de la qualité est reconnue pour être une attitude développée au fil du temps à partir des prestations de la firme, notamment grâce au personnel de contact. La qualité influence positivement le niveau de confiance car elle démontre que les besoins techniques du client peuvent être comblés. La satisfaction est considérée ici à l'aide de la théorie de la disconfirmation affirmant qu'un client satisfait voit ses attentes comblées ou même surpassées ${ }^{15}$. La satisfaction démontre que la cible n'a pas tenté de prendre avantage sur la source et qu'elle est intéressée au bien-être de cette dernière ${ }^{13}$.

L'Historique réfère lui aussi à une expérience antérieure mais en tentant de la quantifier et de relever des expériences vécues, satisfaisantes ou négatives. La durée de la relation influence la confiance puisqu'au fil du temps, des ajustements ont eu lieu et les relations insatisfaisantes ont été abandonnées, laissant place aux relations fonctionnelles ${ }^{14}$. Pour ce qui est des expériences négatives, elles peuvent être occasionnées par des comportements opportunistes de la part de l'autre partie. Ces comportements faisant partie de l'histoire relationnelle laissent des séquelles.

Les deux dernières catégories d'antécédents concernent exclusivement la confiance envers un représentant. L'Orientation-client fait référence à l'évaluation de la personne qui représente l'entreprise à savoir si elle est tournée vers le client. Elle s'opérationnalise surtout par la capacité d'écoute, l'agréabilité de la personne et l'empathie. Ces qualités sont reconnues pour leur influence positive sur la confiance du client, mais aussi sur les résultats de vente.

La dernière catégorie s'intitule Relations interpersonnelles. Ces relations influencent la confiance en la qualifiant à partir d'attributs bien souvent intangibles mais ayant un impact certain. Cette catégorie se réfère à la manière dont se conduisent les relations interpersonnelles et au lien social entre les personnes. La 
fréquence des contacts est un élément important car un acheteur peut observer le comportement du vendeur lors de nombreuses situations et ainsi améliorer son pouvoir prédictif ${ }^{9}$. La connectivité sociale ou affinités entre les individus est importante et se rapporte à la personnalisation et

\section{LES RETOMBÉES}

Les retombées ou conséquences sont des variables dépendantes qui sont significativement influencées par le niveau de confiance. Elles causent peu de dissension dans la littérature et se rassemblent sous trois catégories. Il est à noter que les personnes et les entreprises sont considérées comme poursuivant les mêmes objectifs. C'est pourquoi les catégories des retombées de la confiance sont associées aux deux types de confiance.

La catégorie de retombée qui revient le plus souvent dans la littérature se rapporte à la Continuité de la relation. Cette continuité est l'objectif par excellence du marketing relationnel, où la confiance a une place de choix. Cette approche accorde des ressources à la prolongation de la relation affirmant qu'il est moins coûteux de conserver un client que d'en attirer de nouveaux. Ce sont des bénéfices répartis sur une longue période qui justifient des investissements plus importants à court terme. Le concept d'engagement est notamment une des retombées reconnues de la confiance qui est au cœur du marketing relationnel ${ }^{10}$.

La Recommandation est un construit qui réfère au bouche-à-oreille positif, un échange d'informations entre consommateurs qui ne sont liés à aucun effort marketing. Cette intention du client s'explique probablement de deux manières. Tout d'abord, la réciprocité. Un client qui voit que son vendeur a ses intérêts à cœur, voudra lui rendre la pareille. Une autre explication plausible viendrait une fois de plus de la complexité à établir une relation de confiance. Un client satisfait voudra faire éviter à quelqu'un d'autre la fastidieuse tâche de trouver une cible en qui il peut avoir confiance.

Les Bénéfices relationnels constituent une catégorie plutôt diversifiée. La confiance a à l'adaptation sociale des parties en présence, notamment par l'humour ${ }^{16}$. Aussi, les relations interpersonnelles peuvent être renforcées par le degré de similarité observable (ex. : âge, sexe, taille, façon de d'exprimer) et interne (ex.: intérêts et préférences) entre les parties ${ }^{17}$.

démontré qu'elle pouvait influencer plusieurs composantes d'une relation. La réduction de la tension conflictuelle se rapporte à deux concepts : le conflit fonctionnel et la réduction de conflits. Il y aura toujours certains désagréments dans une relation. Si ces disputes se règlent de façon amicale grâce à un haut niveau de confiance, elles peuvent être une source d'idées, de solutions et donc de conflit fonctionnel ${ }^{10}$. Une autre vision plus conventionnelle du conflit veut que lorsque les parties se font confiance, le nombre de conflits diminue. La confiance est donc considérée comme réduisant la tension conflictuelle.

La réduction de l'incertitude trouve sa raison d'être à plusieurs égards. Tout d'abord, lorsqu'un output est complexe et/ou intangible, il peut être parfois difficile d'évaluer si le choix effectué était le bon. Plusieurs clients peuvent ne pas être familiers avec le produit ou service. Toutefois, il a été démontré qu'un haut niveau de confiance diminuait l'incertitude quant à la prise de décision ${ }^{10}$. Elle servirait donc en quelque sorte d'heuristique. La confiance portée envers l'entreprise ou son représentant devient la base d'évaluation du client lors de sa décision d'achat.

Dans cette étude, la communication est le seul facteur à se retrouver à la fois comme antécédent et comme retombée. Cette situation s'explique par le fait qu'une bonne communication peut faciliter le processus de formation de la confiance notamment grâce à des parties mieux informées ${ }^{14}$. Toutefois, une partie importante de la communication peut se faire de manière informelle et la manière d'entretenir la communication entre deux parties est très discrétionnaire. Une confiance élevée peut accentuer le niveau d'information échangé et faciliter les communications informelles. 
Finalement, la coopération est considérée comme le fait de travailler ensemble afin d'atteindre un but commun ${ }^{10}$. Ce facteur concerne l'étendue que la relation prendra suite à un niveau de confiance important. Il est facile de concevoir que plus une source fait confiance à une cible, plus elle sera encline à développer des

\section{LES MESURES}

Le présent article adopte une vision tridimensionnelle de la confiance. La confiance est donc formée de la bienveillance, de la compétence et de l'intégrité. Toutefois, ces trois dimensions forment un tout et ne doivent pas être conceptualisées de manière séparée.

Bien que plusieurs chercheurs considèrent le construit de la confiance comme multidimensionnel, certaines dimensions ont été démontrées comme étant fortement corrélées entre elles et que leur séparabilité est difficile à prouver. Par exemple, dans une méta-analyse plutôt exhaustive, il n'a pas été possible de prouver qu'il était important de mesurer honnêteté et bienveillance de manière indépendante ${ }^{6}$. Ainsi, les trois dimensions doivent faire partie d'une mesure globale. projets et à élargir sa relation à de nouvelles fonctionnalités. En résumé et selon le cadre conceptuel, la confiance se développe sous l'effet de l'évolution de plusieurs phénomènes et serait à l'origine de plusieurs retombées qu'il convient de procéder à leurs quantifications.

La littérature démontre que les auteurs adaptent souvent les énoncés des échelles au contexte de leur étude. C'est dans cet esprit d'adaptabilité que les tableaux 1 et 2 ont été élaborés ${ }^{18}$. Ils proposent des énoncés à sélectionner afin d'évaluer le niveau de confiance. Idéalement, un minimum de deux énoncés de chaque dimension devrait faire partie de l'échelle utilisée.

En plus des trois dimensions, chaque type de confiance comporte un énoncé d'une catégorie intitulée «Énoncé général ». Sa présence est due à la complexité du construit. L'inclusion d'un tel énoncé permet de contrôler la validité de contenu de l'échelle et de diminuer le biais de désirabilité sociale ${ }^{19}$.

Tableau 1 - Proposition de mesures de la confiance envers l'entreprise

\begin{tabular}{|c|c|}
\hline Dimension & Énoncé \\
\hline \multirow{4}{*}{ Bienveillance } & Cette entreprise a à cœur le succès de notre entreprise. ${ }^{9}$ \\
\hline & L'entreprise fera ce qu'il faut pour nous satisfaire. ${ }^{20}$ \\
\hline & $\begin{array}{l}\text { En cas de problème, l'entreprise déploie des efforts importants pour le } \\
\text { résoudre. }\end{array}$ \\
\hline & En cas de problème, il est facile de faire une réclamation. ${ }^{8}$ \\
\hline \multirow{4}{*}{ Compétence } & $\begin{array}{l}\text { Cette entreprise peut être considérée comme une experte dans son } \\
\text { domaine. }\end{array}$ \\
\hline & Cette entreprise offre un haut niveau de qualité. ${ }^{21}$ \\
\hline & $\begin{array}{l}\text { Cette entreprise a le personnel nécessaire à l'accomplissement de la } \\
\text { tâche. }\end{array}$ \\
\hline & $\begin{array}{l}\text { Cette entreprise a les installations physiques nécessaires à } \\
\text { l'accomplissement de la tâche. }\end{array}$ \\
\hline \multirow{3}{*}{ Intégrité } & Cette entreprise respecte les promesses qu'elle fait. ${ }^{9}$ \\
\hline & Cette entreprise est fondamentalement honnête. ${ }^{9}$ \\
\hline & Les informations que l'entreprise nous transmet sont fiables. ${ }^{23}$ \\
\hline Énoncé général & Cette entreprise est digne de confiance. ${ }^{22}$ \\
\hline
\end{tabular}


Tableau 2 - Proposition de mesures de la confiance envers le représentant

\begin{tabular}{|c|c|}
\hline Dimension & Énoncé \\
\hline \multirow{4}{*}{ Bienveillance } & Le représentant a nos intérêts à cœur. ${ }^{9}$ \\
\hline & Le représentant nous accorde beaucoup d'importance. ${ }^{24}$ \\
\hline & $\begin{array}{l}\text { En cas de problème, le représentant déploie des efforts considérables } \\
\text { pour le résoudre. }^{21}\end{array}$ \\
\hline & $\begin{array}{l}\text { Si nous étions insatisfaits, le représentant montrerait beaucoup de } \\
\text { flexibilité afin que notre relation se poursuive. }\end{array}$ \\
\hline \multirow{4}{*}{ Compétence } & Le représentant est un expert dans le domaine. ${ }^{13}$ \\
\hline & Le représentant est une excellente source d'information utile. ${ }^{25}$ \\
\hline & Le représentant comprend bien notre situation. ${ }^{13}$ \\
\hline & Le représentant répond facilement à nos questions. ${ }^{13}$ \\
\hline \multirow{3}{*}{ Intégrité } & Le représentant respecte les promesses qu'il fait. ${ }^{13}$ \\
\hline & Le représentant a été franc avec nous. ${ }^{9}$ \\
\hline & Les informations données par ce représentant sont fiables. ${ }^{9}$ \\
\hline Énoncé général & Ce représentant est digne de confiance. ${ }^{9}$ \\
\hline
\end{tabular}

\section{DISCUSSION ET CONCLUSION}

\section{La nouvelle typologie proposée devrait permettre d'expliquer un plus fort \\ pourcentage de la variance de la confiance dans les études incluant ce concept.}

Cet article a pour objectif de proposer une nouvelle typologie des antécédents et des retombées de la confiance interentreprises dans un contexte de relation acheteur-vendeur et de proposer une mesure adéquate de la confiance. Les antécédents sont formés par huit catégories et les retombées par trois. La nouvelle typologie proposée devrait permettre d'expliquer un plus fort pourcentage de la variance de la confiance dans les études incluant ce concept. Un des modèles les plus reconnus des antécédents et des retombées de la confiance n'avait permis d'expliquer que $38 \%$ de la variance ${ }^{6}$. Ceci n'est pas surprenant puisque les études tiennent souvent compte uniquement de la confiance envers l'entreprise. En contexte interentreprises, les clients peuvent faire confiance à l'entreprise, mais aussi au représentant et la relation entre ces deux types de confiance est bidirectionnelle9. Ainsi, l'humain doit prendre sa véritable place dans la formation de la confiance et une révision de la typologie des antécédents s'imposait.
La nouvelle typologie d'antécédents est formée de facteurs éprouvés qui devraient contribuer à déterminer plus fortement les niveaux de confiance envers l'entreprise et envers le représentant ainsi que leurs retombées. Cette typologie rassemble deux méta-analyses focalisant chacune sur un des deux types de confiance. La nouvelle typologie se veut intégratrice en rassemblant non seulement les types de confiance, mais aussi en considérant des résultats d'études récentes non contenues dans ces méta-analyses.

Une autre contribution de cet article provient du fait qu'il participe à l'exercice de clarification $\mathrm{du}$ concept de confiance en recensant des visions bien connues et d'autres plus récentes. Une analyse des différentes conceptualisations permet d'établir une vision claire et contemporaine de la confiance à trois composantes. Plusieurs auteurs demandent davantage de recherches théoriques sur la confiance avant de poursuivre l'évaluation empirique du construit ${ }^{2}$. Cet article est donc un pas de plus dans cette démarche théorique de réflexion sur la confiance.

En plus de contribuer à l'élaboration de mesures adéquates de la confiance par le biais de la clarification conceptuelle, cet article propose 
des énoncés reliés aux trois composantes et à une mesure globale du construit pour chaque type de confiance. Cette proposition se base sur des énoncés éprouvés dans la littérature et dont la validité de contenu en lien avec les dimensions mesurées est forte. La rédaction de ces énoncés a été réalisée de manière à assurer leur compatibilité dans plusieurs contextes de relation acheteur-vendeur à l'aide de termes non restrictifs. Leur adaptation à d'autres contextes où la confiance est à l'étude est tout à fait possible étant donné la simplicité des énoncés.

\section{En plus de contribuer à l'élaboration de mesures adéquates de la confiance par le biais de la clarification conceptuelle, cet article propose des énoncés reliés aux trois composantes et à une mesure globale du construit pour chaque type de confiance.}

Les limites de cet article peuvent se traduire en opportunités de recherches futures. Une première limite vient du fait que l'élaboration de la typologie, bien que réalisée à l'aide d'une analyse de contenu de plusieurs études, n'a pas été l'objet d'évaluation empirique ou de tests statistiques. La première étape de spécification du domaine a bien été respectée à l'aide d'une revue extensive de la littérature. Cependant, le modèle doit être validé empiriquement, ce qui pourrait faire l'objet d'un futur projet de recherche.

Avoir les outils pour mesurer la confiance est une chose, avoir les outils pour mesurer ses antécédents et ses retombées en est une autre. L'absence de telles mesures est une autre limite de l'article. Puisque ces deux catégories sont formées de facteurs diversifiés, la décision d'adoption des mesures appropriées demeure difficile. Un exercice rigoureux d'analyse de chaque catégorie, à l'image de ce qui a été fait pour les deux types de confiance, est nécessaire afin de compléter la proposition de mesures contenue dans l'article.

En somme, une vision claire du construit, un modèle intégrant les antécédents et les retombées les plus reconnus de la confiance et une proposition de mesure adéquate du construit sauront certainement trouver écho. Bien que la confiance soit un sujet ayant fait l'objet de plusieurs études, des découvertes récentes permettront de réduire la dissension face à ce construit et d'en venir éventuellement à un consensus sur celui-ci. Cet article pourra servir de tremplin à un processus de recherche plus élaboré sur ce concept si central dans la relation.

\section{BIBLIOGRAPHIE ET NOTES}

${ }^{1}$ Les auteurs aimeraient remercier les évaluateurs anonymes qui ont contribué à l'amélioration de cet article dont une version préliminaire a été publiée dans les actes du colloque «Des relations solides dans un monde en évolution : marketing relationnel et collaboration » organisé dans le cadre du congrès de l'ACFAS 2007 par la Chaire en management des services financiers de l'ESG-UQAM.

${ }^{2}$ Seppänen, Risto, Kirsimarja Blomqvist et Sanna Sundqvist (2007). " Measuring Inter-organizational Trust - a Critical Review of the Empirical Research in 1990-2003 ", Industrial Marketing Management, vol. $36, \mathrm{n}^{\circ} 2$, p. 249-265.

${ }^{3}$ Swan, John E., Michael R. Bowers et Lynne D. Richardson (1999). "Customer Trust in the Salesperson: An Integrative Review and Metaanalysis of the Empirical Literature », Journal of Business Research, vol. 44, $\mathrm{n}^{\circ}$ 2, p. 93-107.

${ }^{4}$ Rousseau, D. M., B.B. Sitkin, R.S. Burt et C. Camerer (1998). " Not so Different After All: A Cross-disciplinary View of Trust», Academy of Management Review, vol. 23, no 3, p. 393-404.

${ }^{5}$ Svensson, Goran (2004). "Vulnerability in Business Relationships: the Gap Between Dependence and Trust », The Journal of Business and Industrial Marketing, vol. 19, $\mathrm{n}^{\mathrm{o}}$ 7, p. 469-483.

${ }^{6}$ Geyskens, Inge, Jan-Benedict E.M. Steenkamp et Nirmalya Kumar (1998). «Generalizations About Trust in Marketing Channel Relationships Using Meta-analysis », International Journal of Research in Marketing, vol. 15, p. 223-48

${ }^{7}$ Lewis, J. David, et Andrew Weigert (1985). « Trust as Social Reality », Social Forces, vol. 63, n 4 , p. 967-985.

${ }^{8}$ Chouk, Inès, et Jean Perrien (2005). « La confiance du consommateur vis-à-vis d'un marchand Internet : Proposition d'une échelle de mesure ", Revue Française de Marketing, vol. 205, $\mathrm{n}^{\circ}$ décembre, p. 5-20. ${ }^{9}$ Doney, P.M., et J.P. Cannon (1997). « An Examination of the Nature of Trust in Buyer-seller Relationships », Journal of Marketing, vol. 62, $\mathrm{n}^{\circ}$ 2, p. 35-51. 
${ }^{10}$ Morgan, Robert M., et Shelby D. Hunt (1994). « The Commitment-trust Theory of Relationship Marketing », Journal of Marketing, vol. 58, $\mathrm{n}^{\circ}$ 3, p. 20-38.

${ }^{11}$ Le terme « représentant » est utilisé dans cet article pour désigner la personne qui représente l'organisation.

${ }^{12}$ Des tableaux présentant les facteurs issus de l'analyse de la littérature liés aux huit catégories d'antécédents et aux trois catégories de retombées sont disponibles auprès des auteurs.

${ }^{13}$ Ganesan, S. (1994). « Determinants of Long-term Relationship Orientation in Buyer-seller Relationship », Journal of Marketing, vol. 58, p. 1-19.

${ }^{14}$ Anderson, Erin, et Barton A. Weitz (1989). « Determinants of Continuity in Conventional Industrial Channel Dyads », Marketing Science, vol. 8, p. 310-23.

${ }^{15}$ Bitner, Mary Jo (1990). « Evaluating Service Encounters: The Effects of Physical Surroundings and Employee Responses », Journal of Marketing Research, vol. 54, $\mathrm{n}^{\mathrm{o}}$ avril, p. 69-82.

${ }^{16}$ Bergeron, Jasmin et Marc-Antoine Vachon (2008). «The Effects of Humour Usage by Financial Advisors in Sales Encounters ", International Journal of Bank Marketing, vol. 26, n ${ }^{\circ}$ 6, 376-398.

${ }^{17}$ Lichtenthal, D.J., et T. Tellefsen (2001). «Toward

a Theory of Business-seller Similarity », Journal of Personal Selling \& Sales Management, vol. 21, $\mathrm{n}^{\circ} 1$, p. 1-14.

${ }^{18}$ Une banque d'énoncés supplémentaires pour évaluer le niveau de confiance est disponible auprès des auteurs.

${ }^{19}$ Blois, Keith J. (1999). « Trust in Business-to-business Relationships: An Evaluation of its Status », Journal of Management Studies, vol. 36, n 2, p. 197-217.

${ }^{20}$ Plank, Richard E., David A. Reid et Ellen Bolman Pullins (1999). "Perceived Trust in Business-tobusiness Sales: A New Measure », Journal of Personal Selling and Sales Management, vol. 19, $\mathrm{n}^{\mathrm{o}} 3$, p. 61-71.

${ }^{21}$ Hess, Jeff, et John Story (2005). «Trust-based Commitment: Multidimensional Consumer-brand Relationships ", The Journal of Consumer Marketing, vol. 22, $\mathrm{n}^{\mathrm{o}}$ 6, p. 313-22.

${ }^{22}$ Gounaris, Spiros P. (2005). « Trust and Commitment Influences on Customer Retention : Insights from Business-to-business Services », Journal of Business Research, vol. 58, p. 126-140.

${ }^{23} \mathrm{Graf}$, Raoul (2004). Le rôle médiateur de la confiance dans les relations acheteur-vendeur : Une application à la relation entreprise fondée sur la connaissance directeur de comptes bancaires, Thèse de doctorat, Université du Québec à Montréal, Montréal.

${ }^{24}$ Sirdeshmukh, Deepak, Jagdip Singh et Barry Sabol (2002). « Consumer Trust, Value, and Loyalty in Relational Exchanges", vol. 66, no 1, p. 15-37.

${ }^{25}$ Swan, John E., Fred I. Trawick Jr., David R. Rink et Jenny J. Roberts (1988). « Measuring Dimensions of
Purchaser Trust of Industrial Salespeople", Journal of Personal Selling and Sales Management, vol. 8, $\mathrm{n}^{\mathrm{o}} 1$, p. $1-9$. 


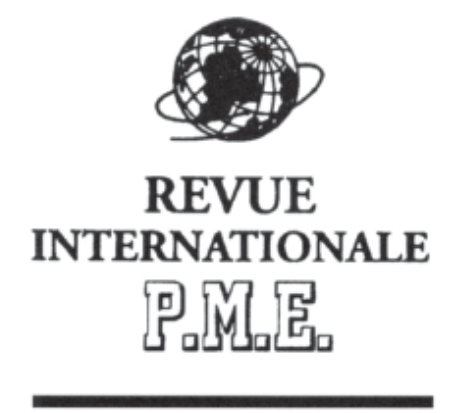

Volume 24, numéro 2, 2011

\title{
SOMMAIRE
}

\author{
ÉDITORIAL \\ Louis Raymond
}

\section{ARTICLES}

Le soutien public aux projets innovants des PME : une évaluation de deux territoires, en Italie et en France Secondo Rolfo et Serena Novero

$$
\text { Ceris-CNR }
$$

Les entreprises à internationalisation précoce et rapide :

étude d'un cas exploratoire par le Business Model

Vinciane Servantie

Facultad de Administración - Universidad de Los Andes - Colombia et Équipe de recherche en entrepreneuriat - IRGO - Université de Bordeaux - France

Prendre les rênes de la PME familiale et se faire accepter Vivi Koffi et Jean Lorrain

Université de Moncton et Université du Québec à Trois-Rivières

L'entrepreneur comme clé de voûte de l'internationalisation rapide de son entreprise Mathieu Cabrol et Véronique Favre-Bonté GSCM Montpellier Business School et Université de Savoie (IAE Savoie Mont-Blanc), IREGE

Les dispositifs de gestion des compétences dans les PME : mythe ou réalité ? Étude auprès de 28 digireants de PME Jocelyne Abraham, Franck Brillet,Patricia Coutelle et Annabelle Hulin IAE de Tours - Université de Tours

École polytechnique universitaire de Tours - Université de Tours

Comprendre les pratiques d'entreprises en matière d'intrapreneuriat : une proposition de caractérisation

Olivier Lisein et Julie Degré

LENTIC - HEC-École de gestion de I'Université de Liège

COMPTES RENDUS

INFORMATIONS

TABLES DES MATIĖRES DES REVUES ASSOCIÉES 